\title{
Sub-Conjunctival Injection of Antibiotics vs. Povidone-Iodine Drop on Bacterial Colonies in Phacoemulsification Cataract Surgery
}

\author{
Mahamoudreza Panahibazaz ${ }^{1}$; Mojataba Moosavian ${ }^{2}$; Gholamreza Khataminia ${ }^{1}$; Mostafa \\ Feghhi $^{1,} ;$ Farsim Yazdi $^{1} ;$ Effat Abbasi Montazeri $^{2}$ \\ ${ }^{1}$ Department of Ophthalmology, Ophthalmic Research Center, Imam Khomeini Hospital, Ahvaz Jundishapur University of Medical Sciences, Ahvaz, IR Iran \\ ${ }^{2}$ Department of Microbiology, Infectious Research Center, Ahvaz Jundishapur University of Medical Sciences, Ahvaz, IR Iran \\ ${ }^{*}$ Corresponding author: Mostafa Feghhi, Department of Ophthalmology, Imam Khomeini Hospital, Ahvaz Jundishapur University of Medical Sciences, Ahvaz, IR Iran. Tel: +98- \\ 6113738383, Fax:+98-6113367562, E-mail: feghhim671@live.com
}

Received: June 25, 2013; Revised: November 10, 2013; Accepted: March 31, 2014

\begin{abstract}
Background: Postoperative endophthalmitis is one the most serious complications of cataract surgery. The majority of causative organisms in this destructive infection come from the patient's own periocular flora. Efforts have been made to reduce the virulence of organisms in the eyelid and conjunctiva with perioperative topical antibiotics, preparation of surgical field, covering eyelids and conjunctival surface with $5 \%$ povidone-iodine solution and intracameral antibiotics at the time of surgery to minimize the risk of endophthalmitis.

Objectives: We assessed the effect of subconjunctival injection of cefazolin and pouring povidone-iodine on the conjunctiva bacterial colony forming units (CFU) in phacoemulsification cataract surgery.

Patients and Methods: In this prospective, randomized, double-blind clinical trial, 122 patients having phacoemulsification cataract surgery with clear corneal incision and topical anesthesia were randomized into two groups including group1(subconjunctival injection of cefazolin) and group 2 (recipients of a drop of povidone-iodine). Cultures were collected from the bulbar conjunctiva at the injection site and from the corresponding location in the patient's eye, three different times.

Results: The mean of eyelid samples on blood and chocolate agars, on the day after compared to the day before the surgery in group 1 showed a $52 \%$ and $56 \%$ reduction. These values were $58 \%$ and $50 \%$ in group $2(\mathrm{P}<0.05)$. The mean CFU of conjunctiva before and at the end of surgery on blood and chocolate agars showed $57 \%$ and $56 \%$ reduction in group one and $51 \%$ and $52 \%$ reduction in group $2(\mathrm{P}<0.05)$. While comparing mean CFU of conjunctiva at the end and one day post-surgery (interval of $14 \pm 2$ hours) showed $27 \%$ and $27 \%$ increase in group 1 and $20 \%$ and $21 \%$ increase in group $2(\mathrm{P}<0.05)$, which reflects conjunctival flora proliferation during the early postoperative period.

Conclusions: Due to the good tolerance of patients towards topical anesthesia, pouring a drop of povidone-iodine $10 \%$ seems to be a simple and acceptable method to reduce the growth of microorganisms of the conjunctiva.
\end{abstract}

Keywords:Injection; Cefazolin; Povidone-Iodine; Phacoemulsification

\section{Background}

Cataract surgery is one of the most common ophthalmic surgical procedures currently performed and has become one of the most prevalent surgeries because of the growth of the elderly population $(1,2)$. Postoperative endophthalmitis is one the most serious complications of cataract surgery that always presents a poor visual prognosis (1-8). Despite the low incidence rate of endophthalmitis (0.03-0.015), large numbers of cataract surgeries contribute to this low rate and thus preventive measures are important $(3,6,8,9)$. Several studies have shown that the eyelids and conjunctiva are the source and the origin of endophthalmitis following cataract surgery (3, 4, 6-8).

Phacoemulsification using topical anesthesia is at present the most common cataract surgery technique (10-13). In this method, the small incision may not be completely closed by stromal hydration and a potential space may remain between anterior the chamber and cul-de-sac for hours after surgery (9-13). Consequently, the elimination or reduction of microorganisms contained in the surgical field requires deliberate attention. The belief that the organism responsible for endophthalmitis may enter the eye, during surgery (10-13), has led to the use of antibiotics as infusion or injection within the anterior chamber (AC) (1,14-18), although minute variation error in antibiotics concentration may induce endothelial cell decompensation and irreversible corneal edema $(4,15)$. Increasing injection of antibiotics within the AC following cataract surgery, especially after the European Society Cataract and Refractive Surgery(ESCRS) study, has led to decreased postoperative endophthalmitis by about five folds compared to the control group $(1,14$, 16). However, the use of topical levofloxacin and third and fourth generation fluoroquinolones $(1,15)$ for up to

Copyright (C) 2014,Ahvaz Jundishapur University of Medical Sciences; Published by Kowsar. This is an open-access article distributed under the terms of the Creative Commons Attribution-NonCommercial 4.0 International License (http://creativecommons.org/licenses/by-nc/4.0/) which permits copy and redistribute the material just in noncommercial usages, provided the original work is properly cited. 
one week after surgery, may still allow the transmission of the organism from the conjunctiva and eyelid, especially during the first post-operative day, which means there remains a strong possibility for endophthalmitis $(7,9,11)$. At present, among different methods of preoperative prophylaxis, applying $10 \%$ povidone-iodine to prepare the skin of the eyelid and use a $5 \%$ povidoneiodine with or without third and fourth generation fluoroquinolones, are the most acceptable methods that reduce cul-de-sac microbial colonies and possibility of endophthalmitis $(2,5-8,10,12,14,16,17)$. However, there is no consensus about proper methods for post cataract surgery.

\section{Objectives}

Thus, in this study we assessed the effect of subconjunctival injection of cefazolin and placing one drop of $10 \%$ povidone-iodine in the conjunctival sac on the bacterial colony forming units at completion of surgery until the first day post-surgery.

\section{Patients and Methods}

\subsection{Study design and Population}

This double-blinded, randomized clinical trial was conducted on 122 patients, who had undergone cataract surgery at Imam Khomeini Hospital since October 2011. This study was approved by Ahvaz Jundishapur University of Medical Sciences ethics committee and an informed consent was obtained from all patients.

\subsection{Drug}

Povidone-iodine (5\% and 10\%) was purchased from IRAN Najo pharmaceutical Co., Tehran, Iran. Cefazolin was obtained from Jaber Ebre Hayyan pharmaceutical MFG Co, Tehran, Iran.

\subsection{Inclusion and Exclusion Criteria}

Ophthalmic patients without active infection were included. Subjects who were using topical or systemic antibiotics, had a history of sensitivity to iodine, a history of previous eye surgery, pregnant women and those who refused to participate were excluded.

\subsection{Intervention}

Patients were randomly divided in to two groups, including group 1 (cefazolin injection under the conjunctiva at the end of surgery) and group 2 (povidone-iodine recipients at the end of surgery). In both groups, before the preparation of the operation filed, two samples from each eyelid, conjunctiva, and the fornix were collected using sterile swabs impregnated with sterile distilled water. The samples were then aseptically inoculated using the linear method to blood and chocolate agar media to determine bacterial colony counts. After preparing the patient's skin, their cheeks and forehead were washed using povidone-iodine $10 \%$, their eyelids and eye lashes were covered with a sterile adhesive, and next to where the speculum was placed, the edge of the eyelids were washed using swabs emulsified in povidone-iodine10\% and all patients received povidone iodine $5 \%$ in the conjunctival cul-de-sac for 3 minutes before the operation.

All cataract surgeries were performed according to standard methods of phacoemulsification, through the temporal clear corneal incision by two surgeons, also operating at the Imam Khomeini Hospital. After finishing the surgery, another sampling of the conjunctiva and the fornix was performed, using the above mentioned method. In Group 1, 50 mg of cefazolin (0.5 CC of $100 \mathrm{mg}$ / $\mathrm{mL}$ ) was injected under the conjunctiva and in Group 2, a drop of povidone-iodine 10\% was applied on the conjunctival sac. The patient's eye was covered by a bandage patched and protected by a plastic shield and the collected samples were sent to the laboratory incubator.

The day after the surgery (with a time interval of $14 \pm 2$ hours from termination of surgery) after removing the bandage, two samples were collected from the eyelid and conjunctival sac and inoculated in the aforementioned manner and sent to the laboratory. Besides, the blood agar plate was incubated at $37^{\circ} \mathrm{C}$ under anaerobic conditions and the chocolate agar plate was incubated in $5 \% \mathrm{CO}_{2}$ at $37^{\circ} \mathrm{C}$; all plates were incubated for 24 to 48 hours. All plates were examined macroscopically for the presence or absence of bacterial colonies. The colony counts have performed at each time point according to the colony-forming units (CFU). Following two days of incubation, each specimen was analyzed by the laboratory scientist, and when colonies were present, basic bacteriology identification tests were performed to identify the present bacteria. In this, study antibiotic drops were not administrated for the days before and at the end of the surgery.

\subsection{Statistical Analysis}

Data were analyzed using the SPSS 15.0 software. Due to the highly abnormal distribution of data, the use of nonparametric tests was preferred over parametric tests. The Wilcoxon test was used to compare the observed count in each group and the Mann-Whitney test was used to compare colony count between the two groups. To determine the effect of povidone-iodine $10 \%$ solution and antibiotics at the end of the cataract surgery, conjunctival colony count and confidence limits of $95 \%$, test power of $80 \%$ and sample size for $\mathrm{P} \leq 0.05$, were calculated.

\section{Results}

During this project, data were collected from 122 eyes of 122 patients with cataract. In group 1 ( 61 recipients of subconjunctival cefazolin), there were 34 (55.7\%) males and 27 (43.3\%) females, and in group 2 (61 recipients of 
povidone-iodine), there were $33(54.1 \%)$ males and 28 (45.9\%) females. The age range of patients in group 1 was 34-84 years, with a mean of 68.1 years, and in group 2 the range was 35-84 years with a mean of 67.2 years $(\mathrm{P}<$ 0.05). The mean rank of colony count of eyelid samples on blood and chocolate agars on the day after surgery in groups 1 showed 52\% and 56\% reduction and in second group reduction was 58\% and 50\% respectively $(\mathrm{P}<0.05)$ compared to the day before surgery (Table 1 ).

In Group 1 the median blood and chocolate agar colony counts changed from 100,000 preoperatively to 100 during the postoperative day, indicating a $99.9 \%$ reduction. In group 2 the median blood agar colony count decreased from 100,000 preoperatively to 290 during the postoperative day indicating a $99.7 \%$ reduction. These values regarding chocolate agar colony counts changed from 50,000 to 100 , which indicates a reduction of about $99.8 \%$. Comparing the colony counts of conjunctiva samples during the pre-operative and first postoperative days, in group 1 the mean rank of colony count blood and chocolate agars showed $48 \%$ and $45 \%$ reduction and in group 2 a $44 \%$ and $40 \%$ reduction was indicated, respectively; these results indicate a significant decline in colony counts on the first postoperative day compared to the preoperative day, for both groups $(\mathrm{P}<0.05)$ (Table 1). However, the difference between the two groups was not significant $(\mathrm{P}>0.1)$ (Table 3$)$.

Between group comparisons of the colony counts of conjunctiva samples pre-surgery and at the end of surgery, was also done. In group 1, the mean rank of colony count blood and chocolate agars were $57 \%$ and $56 \%$, and in group 2 these values were, 51\% and 52\%, respectively, showing a significant reduction, in colony counts of conjunctiva samples at the end of surgery compared to preoperative samples, in both groups $(\mathrm{P}<0.05)$ (Table 2) Mann-Whitney tests proved that this difference in the two groups is not statistically significant $(P>0.1)$ (Table 3). By comparing the colony counts of conjunctiva samples at the end of the surgery and the day after surgery, it was determined that in group 1 the mean rank of both blood and chocolate agars showed $27 \%$ and in group 2 these showed $20 \%$ and $21 \%$ increase, respectively, which reflects the proliferation of bacteria in the conjunctival crypts at the interval between the day after surgery compared to the end of surgery in both group $(\mathrm{P}<0.05)$ (Table 2). However, these differences between the two groups were not statistically significant $(\mathrm{P}>0.1)$ (Table 3).

Table 1. Mean Rank of Eyelids and Conjunctival Samples on the day Before and the day After Surgery ${ }^{\mathrm{a}}$

\begin{tabular}{|c|c|c|c|c|c|c|}
\hline \multirow[b]{2}{*}{ Group } & \multicolumn{3}{|c|}{ Eyelid Sample Day Before and After Surgery } & \multicolumn{3}{|c|}{ Conjunctival Sample Day Before and After Surgery } \\
\hline & & $\mathbf{n}$ & Mean Rank & & $\mathbf{n}$ & Mean Rank \\
\hline \multirow[t]{8}{*}{1} & L. ba. P-L. ba. b Neg. Rank & $43^{\mathrm{b}, \mathrm{c}}$ & 29.80 & C. ba.p-C. ba. b Neg. Rank & $35^{\mathrm{b}, \mathrm{c}}$ & 31.84 \\
\hline & Pos. Rank & $6^{d}$ & 14.17 & Pos. Rank & $13^{\mathrm{d}}$ & 16.52 \\
\hline & Ties & $11^{\mathrm{e}}$ & - & Ties & $12^{\mathrm{e}}$ & \\
\hline & Total & 60 & - & Total & 60 & \\
\hline & L. ch. p-L. ch. b Neg. Rank & $44^{\mathrm{b}, \mathrm{c}}$ & 30.38 & C. ch.p-C. ch. b Neg. Rank & $30^{\mathrm{b}, \mathrm{c}}$ & 29.42 \\
\hline & Pos. Rank & $3^{\mathrm{d}}$ & 13.33 & Pos. Rank & $18^{\mathrm{d}}$ & 16.15 \\
\hline & Ties & $13^{\mathrm{e}}$ & & Ties & $12^{\mathrm{e}}$ & \\
\hline & Total & 60 & & Total & 60 & \\
\hline \multirow[t]{8}{*}{2} & L. ba. p-L. ba. b Neg. Rank & $40^{\mathrm{b}}$ & 31.09 & C. ba.p-C. ba. b Neg. Rank & $35^{\mathrm{b}, \mathrm{c}}$ & 31.84 \\
\hline & Pos. Rank & $8^{\mathrm{d}}$ & 12.94 & Pos. Rank & $13^{\mathrm{d}}$ & 16.52 \\
\hline & Ties & $12^{\mathrm{e}}$ & & Ties & $12^{\mathrm{e}}$ & \\
\hline & Total & 60 & & Total & 60 & \\
\hline & L. ch. p-L. ch. b Neg. Rank & $43^{b, d}$ & 31.60 & C. ch. p-C. ch. b Neg. Rank & $30^{\mathrm{b}, \mathrm{c}}$ & 29.42 \\
\hline & Pos. Rank & $6^{\mathrm{d}}$ & 15.83 & Pos. Rank & $18^{\mathrm{d}}$ & 16.15 \\
\hline & Ties & $11^{\mathrm{e}}$ & & Ties & $12^{\mathrm{e}}$ & \\
\hline & Total & 60 & & Total & 60 & \\
\hline
\end{tabular}


Panahibazaz Met al.

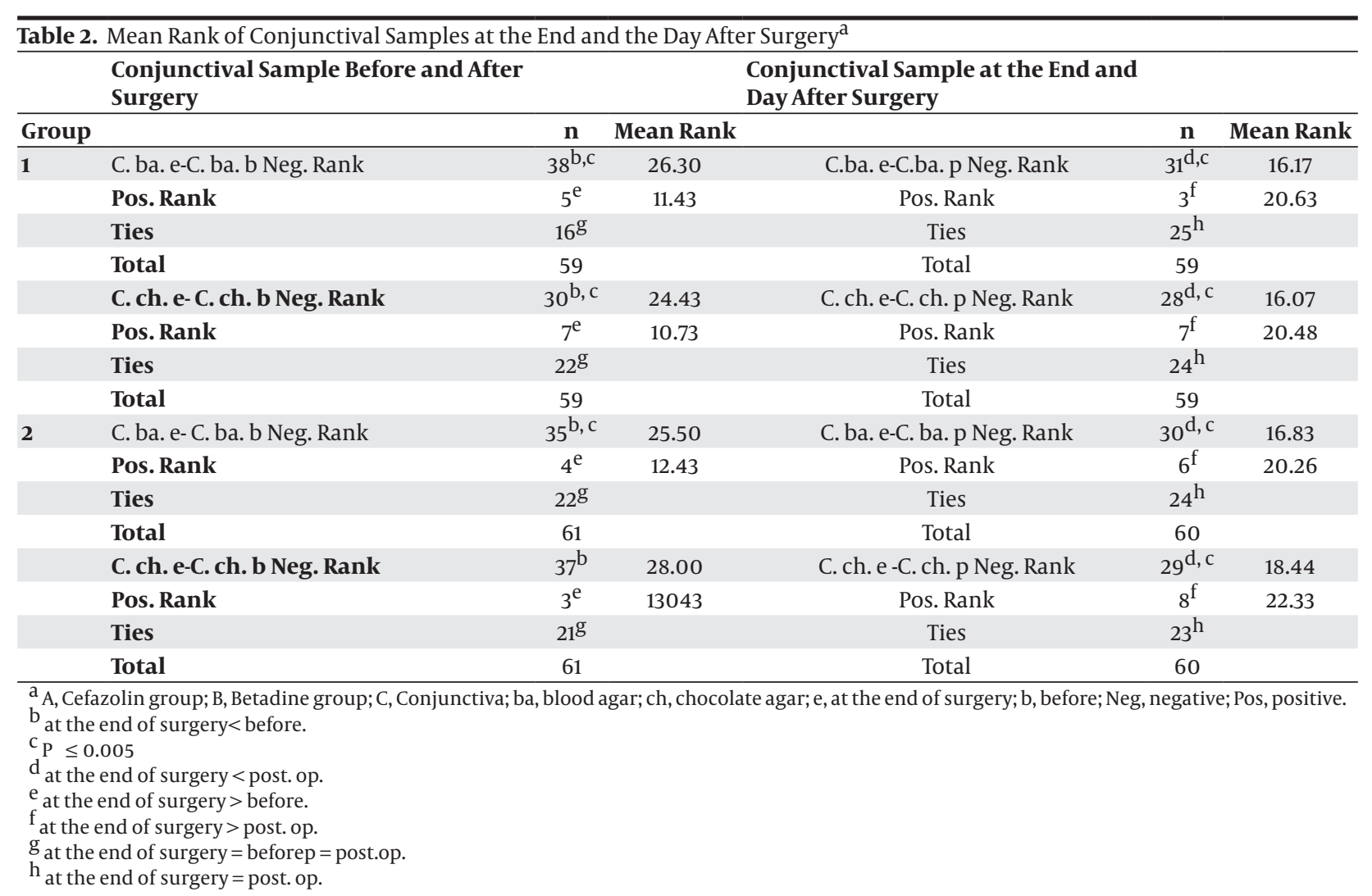

Table 3. Comparison of Blood Agar and Chocolate Agar Samples From Groups 1 and $2^{\mathrm{a}}$

\begin{tabular}{|c|c|c|c|}
\hline & $\mathbf{n}$ & Mean Rank & PValues \\
\hline \multicolumn{4}{|c|}{$\begin{array}{l}\text { Eyelid sample day before and } \\
\text { after surgery }\end{array}$} \\
\hline L. ba. diff 1 & 60 & 62.67 & \multirow[t]{2}{*}{0.49} \\
\hline L. ba. diff 2 & 60 & 58.33 & \\
\hline L. ch. diff 1 & 60 & 65.62 & \multirow[t]{2}{*}{0.106} \\
\hline L.ch. diff 2 & 60 & 55.38 & \\
\hline \multicolumn{4}{|c|}{$\begin{array}{l}\text { Conjunctival sample day be- } \\
\text { fore and after surgery }\end{array}$} \\
\hline C.ba.diff 1 & 60 & 52.88 & \multirow[t]{2}{*}{0.251} \\
\hline C.ba.diff 2 & 60 & 64.13 & \\
\hline C.ch.diff1 & 60 & 58.96 & \multirow[t]{2}{*}{0.626} \\
\hline C.ch.diff 2 & 60 & 62.04 & \\
\hline \multicolumn{4}{|c|}{$\begin{array}{l}\text { Conjunctival sample before } \\
\text { and after surgery ( delete day) }\end{array}$} \\
\hline C.ba. diff 1 & 59 & 57.17 & \multirow[t]{2}{*}{0.291} \\
\hline C.ba. diff 2 & 61 & 63.72 & \\
\hline C.ch. diff 1 & 59 & 59.07 & \multirow[t]{2}{*}{0.643} \\
\hline C.ch. diff 2 & 61 & 61.89 & \\
\hline \multicolumn{4}{|c|}{$\begin{array}{l}\text { Conjunctival sample at the end } \\
\text { and day after surgery }\end{array}$} \\
\hline C.ba diff 1 & 59 & 61.99 & \multirow[t]{2}{*}{0.520} \\
\hline C.ba diff 2 & 60 & 58.04 & \\
\hline C.ch $\operatorname{diff} 1$ & 59 & 62.97 & \multirow[t]{2}{*}{0.340} \\
\hline C.ch $\operatorname{diff} 2$ & 60 & 57.08 & \\
\hline
\end{tabular}

\section{Discussion}

The primary source of postoperative endophthalmitis, are bacteria present in the eyelids and conjunctiva. Thus eliminating or reducing these microorganisms can decrease the risk of endophthalmitis (3). The clear corneal incision is the most common method in phacoemulsification surgery. Numerous reports have indicated (9-13) that even through completely sealed incisions, bacteria available in tears and the conjunctiva sac may possibly enter the eye in the early postoperative hours, which can lead to increased frequency of postoperative bacterial endophthalmitis (9-13). Taban and colleagues (9) showed that as India ink can cross the seemingly closed incision of the clear cornea and enter the anterior chamber, bacteria can also get into the eye.

Light micrographs that were obtained from the clear corneal incision without suture, revealed that India ink could penetrate across the incision. This phenomenon indicates that before any process of wound healing, tears and microorganisms can enter into the anterior chamber in the early stages after surgery, so the integrity of surgical incision is a crucial factor in postoperative bacterial endophthalmitis prevention. Many reports have demonstrated (9-12) the relationship between corneal incision diffects without suture and increasing frequency of postoperative bacterial endophthalmitis (9-12). In most cases the end of surgery incision is self-sealed or completely water tight after stromal hydration (9-12), however wound integrity is influenced by changes in intra-ocular pressure (IOP). A report 
showed (9) that $21 \%$ of eyes following phacoemulsification surgery from the clear corneal incision had IOP of $5 \mathrm{mmHg}$ or less, which is a temporary hypotony in the first 24 hours and may allow the conjunctival microorganisms access the anterior chamber of the eye through the incision.

In vitro studies showed that tears and cul-de-sac content can enter the anterior chamber via single or two planed clear corneal incision(9-13). Eye movements and eyelid squeezing in the immediate period after surgery with local anesthesia changes the IOP and transient wound gap, which facilitates bacterial entrance in to the eye (8-11). Fluoroquinolones drops are prophylactic agents for the ocular condition before intraocular surgeries. The purpose of prophylactic use of antibiotics before cataract surgery is reducing pathogenic microorganisms in the eyelids and conjunctiva, and obtaining proper concentrations of antibiotics in the cornea and Aqueous humor $(19,20)$. Thus, for the antibiotic to be effective it must not only have high tissue penetration but it should also be capable of eliminating conjugative bacteria (19).

Bucci and colleagues (21) evaluated the ocular surface and aqueous antimicrobial effects of gatifloxacin and moxifloxacin administered in two dosing regimens on the normal florabacteria of patients undergoing phacoemulsification. The authors claimed that these two antibiotics equally reduced the chances of getting a positive culture of the organism from the aqueous liquid. Vasavada et al. conducted a prospective randomized triple-masked trial, including two parts, in which one part evaluated the aqueous concentration of moxifloxacin following two dosing regimens of topically administered moxifloxacin hydrochloride ophthalmic solution $0.5 \%$ (vigamox) (19); while the second part determined whether a regimen of vigamox administered on the day of cataract surgery reduces conjunctival bacterial flora. They claimed that both regimens produced substantially higher aqueous concentrations; topical moxifloxacin administered two hours before surgery achieved significantly higher aqueous concentrations. Other studies $(1,2,17)$ were conducted on the efficacy of levofloxacin and showed that topical application of this antibiotic accompanied by washes with povidone-iodine, results a more effective reduction in bacterial ocular surface.

Another factor that effects endophthalmitis after phacoemulsification is incision diffect, Maxwell and colleagues showed (9) that $80 \%$ of postoperative bacterial endophthalmitis were related to incision diffects such as a wound gape and malposition. Simultaneous suture handling of corneal incision with application of povidoneiodine at closure, and initiation of antibiotic eye drops within the first 24 hours of surgery can reduce the risk of endophthalmitis (10). Thus far, there has not been the possibility to create a sterile conjunctivitis (5). The effect of $5 \%$ povidone-iodine as a broad spectrum antibiotic to reduce microbial flora of the conjunctiva and eyelids and decrease the incidence of endophthalmitis has been proven by many studies $(1,2,5-10,14-17,19,20)$. Although, the use of topical antibiotics one hour before or during the day of the surgery reduces the microbial flora of the conjunctiva and eyelids, yet it does not eliminate the bacteria from the surgical field. Thus, the growth of organisms during the first hours after the surgery and the possibility of getting into the anterior chamber via surgical incision remains a potential risk for endophthalmitis $(7,8)$. We tried using subconjunctival injection of cefazolin and povidone-iodine $10 \%$ to control microorganism replication during the early hours after surgery.

This study assessed the effect of post-cataract surgery conjunctival injection of cefazolin and 10\% povidone-iodine on bacterial colony count at end of the surgery until the first post-surgery day, and showed no statistical difference between usage of povidone-iodine and antibiotics. Subconjunctival injection of antibiotics at the end of cataract surgery is one of the oldest prophylactic measures to prevent endophthalmitis (6). But later studies showed that subconjunctival injection was not effective in prevention of endophthalmitis (7). The effect of povidone-iodine as a broad spectrum antiseptic to reduce the incidence of endophthalmitis in the preoperative preparation period, has been reported by many studies $(5-8,10-12,14,16,17)$, which showed the use of povidone-iodine $5 \%$ in the fornix before the surgery, significantly decreases conjunctival colonies. Feghhi and colleagues approved the efficacy of povidone-iodine on corneal ulcers compared with standard antibiotics in animals (22). Thus, the most acceptable method for preparation of the eyelid and conjunctiva, is the use of povidone-iodine, however the preferred method for the end of the surgery still depends on the surgeon's opinion (14-16, 18-20, 23).

In this study we showed that the use of povidone-iodine before surgery is very effective in reducing conjunctival bacteria counts during the operation; eyelid mean colony counts on blood and chocolate agar decreased from 100,000 on the preoperative day to 100 on the postoperative day indicating about a $99.9 \%$ reduction rate. These values in the group that received subconjunctival antibiotics at the end of the surgery (Group A), decreased from 100,000 on the preoperative day to 290 on the days after the surgery showing about a $99.7 \%$ reduction $(\mathrm{P}>0.1)$. However, the colony count of days after the surgery was significantly higher than that of the end of the surgery, in both study groups. Comparison of colony counts of the conjunctiva at the end of the surgery and on the first day after the operation for group 1, showed an increase of $27 \%$ and group 2, showed $20 \%$ and $21 \%$ increase; although in terms of numeric values, use of povidone-iodine, at the end of surgery was associated with less bacterial colony replication on the first day after the surgery, yet statistical comparisons showed no significant differences between the two groups $(\mathrm{P}>0.1)$. This implies that the use of subconjunctival cefazolin and povidone-iodine at the end of the surgery, will continue reducing bacterial counts from the time of preparation before the surgery until the day after the operation. The reason is that despite all arrange- 
ments and preparations, a completely sterile conjunctiva in the fornices is probably unreachable, because this area has deep crypts, which despite using povidone-iodine preoperatively, complete sterility among these crypts is not possible (5). Therefore, these residual values can grow in the interval between end of the surgery and the next day, and if no antiseptic agents are used at the end of the surgery, they may grow more and make further colonies that finally increase the risk of endophthalmitis.

It is important to mention that in none of the 122 patients, acute or chronic postoperative bacterial endophthalmitis was observed, and those patients, who were exposed to povidone-iodine $10 \%$, tolerated this treatment well and no complications and adverse effects such as corneal edema or epithelial diffects, and sensitivity to povidone-iodine, were detected. In conclusion, due to the ease and cost efficiency of povidone-iodine $10 \%$ and patients good tolerance of this treatment, when the surgeon does not intended to use injected intracameral antibiotic at the end of the cataract surgery, pouring a drop of povidone-iodine 10\% seems to be a simple and acceptable method to reduce the growth of microorganisms of the conjunctiva.

\section{Acknowledgements}

None Declare.

\section{Authors' Contributions}

Study concept and design: Mahamoudreza Panahibazaz. Acquisition of data: all authors. Analysis and interpretation of data:Mahamoudreza Panahibazaz. Mojataba Moosavian. Farsim Yazdi. Drafting of the manuscript: Farsim Yazdi. Mahamoudreza Panahibazaz. Critical revision of the manuscript for important intellectual content: Gholamreza Khataminia. Mostafa feghhi. Mahamoudreza Panahibazaz. Statistical analysis: Mahamoudreza Panahibazaz. Farsim Yazdi. Administrative, technical, and material support: Mostafa feghhi. Mojataba Moosavian. Effat Abbasi-Montazeri

\section{Financial Disclosure}

All authors declare that they have no conflict of interest.

\section{Funding Support}

This study was granted by a research affairs grant (Grant U-86037).

\section{References}

1. Endophthalmitis Study Group ESOC, Refractive S. Prophylaxis of postoperative endophthalmitis following cataract surgery: results of the ESCRS multicenter study and identification of risk factors. J Cataract Refract Surg. 2007;33(6):978-88.

2. Seal D, Reischl U, Behr A, Ferrer C, Alio J, Koerner RJ, et al. Laboratory diagnosis of endophthalmitis: comparison of microbiology and molecular methods in the European Society of Cataract \& Refractive Surgeons multicenter study and susceptibility testing.J Cataract Refract Surg. 2008;34(9):1439-50.
3. Pathengay A, Flynn HW, Jr, Isom RF, Miller D. Endophthalmitis outbreaks following cataract surgery: causative organisms, etiologies, and visual acuity outcomes. J Cataract Refract Surg. 2012;38(7):1278-82.

4. Yoeruek E, Spitzer MS, Saygili O, Tatar O, Biedermann T, Yoeruek $\mathrm{E}$, et al. Comparison of in vitro safety profiles of vancomycin and cefuroxime on human corneal endothelial cells for intracameral use. J Cataract Refract Surg. 2008;34(12):2139-45.

5. Mino de Kaspar H, Chang RT, Singh K, Egbert PR, Blumenkranz MS Ta CN. Prospective randomized comparison of 2 different methods of $5 \%$ povidone-iodine applications for anterior segment intraocular surgery. Arch Ophthalmol. 2005;123(2):161-5.

6. Buzard K, Liapis S. Prevention of endophthalmitis. J Cataract Refract Surg. 2004;30(9):1953-9.

7. Ciulla TA, Starr MB, Masket S. Bacterial endophthalmitis prophylaxis for cataract surgery: an evidence-based update. Ophthalmology. 2002;109(1):13-24.

8. Leong JK, Shah R, McCluskey PJ, Benn RA, Taylor RF. Bacterial contamination of the anterior chamber during phacoemulsification cataract surgery. J Cataract Refract Surg. 2002;28(5):826-33.

9. Taban M, Sarayba MA, Ignacio TS, Behrens A, McDonnell PJ. Ingress of India ink into the anterior chamber through sutureless clear corneal cataract wounds. Arch Ophthalmol. 2005;123(5):643-8.

10. Thoms SS, Musch DC, Soong HK. Postoperative endophthalmitis associated with sutured versus unsutured clear corneal cataract incisions. BrJ Ophthalmol. 2007;91(6):728-30.

11. Herretes S, Stark WJ, Pirouzmanesh A, Reyes JM, McDonnell PJ, Behrens A. Inflow of ocular surface fluid into the anterior chamber after phacoemulsification through sutureless corneal cataract wounds. Am JOphthalmol. 2005;140(4):737-40.

12. Garcia-Arumi J, Fonollosa A, Sararols L, Fina F, Martinez-Castillo V, Boixadera A, et al. Topical anesthesia: possible risk factor for endophthalmitis after cataract extraction. J Cataract Refract Surg. 2007;33(6):989-92.

13. Gajjar D, Praveen MR, Vasavada AR, Pandita D, Vasavada VA, Patel $\mathrm{DB}$, et al. Ingress of bacterial inoculum into the anterior chamber after bimanual and microcoaxial phacoemulsification in rabbits. J Cataract Refract Surg. 2007;33(12):2129-34.

14. Barreau G, Mounier M, Marin B, Adenis JP, Robert PY. Intracameral cefuroxime injection at the end of cataract surgery to reduce the incidence of endophthalmitis: French study.JCataract Refract Surg. 2012;38(8):1370-5.

15. Espiritu CR, Caparas VL, Bolinao JG. Safety of prophylactic intracameral moxifloxacin $0.5 \%$ ophthalmic solution in cataract surgery patients. J Cataract Refract Surg. 2007;33(1):63-8.

16. Lane SS, Osher RH, Masket S, Belani S. Evaluation of the safety of prophylactic intracameral moxifloxacin in cataract surgery. J Cataract Refract Surg. 2008;34(9):1451-9.

17. Chang DF, Braga-Mele R, Mamalis N, Masket S, Miller KM, Nichamin LD, et al. Prophylaxis of postoperative endophthalmitis after cataract surgery: results of the 2007 ASCRS member survey. J Cataract Refract Surg. 2007;33(10):1801-5.

18. Romero P, Mendez I, Salvat M, Fernandez J, Almena M. Intracameral cefazolin as prophylaxis against endophthalmitis in cataract surgery. J Cataract Refract Surg. 2006;32(3):438-41.

19. Vasavada AR, Gajjar D, Raj SM, Vasavada V, Vasavada V. Comparison of 2 moxifloxacin regimens for preoperative prophylaxis: prospective randomized triple-masked trial. Part 1: aqueous concentration of moxifloxacin.J Cataract Refract Surg. 2008;34(8):1379-82.

20. Ong-Tone L. Aqueous humor penetration of gatifloxacin and moxifloxacin eyedrops given by different methods before cataract surgery. J Cataract Refract Surg. 2007;33(1):59-62.

21. Bucci FA, Jr, Amico LM, Evans RE. Antimicrobial efficacy of prophylactic gatifloxacin $0.3 \%$ and moxifloxacin $0.5 \%$ in patients undergoing phacoemulsification surgery. Eye Contact Lens. 2008;34(1):39-42.

22. Feghhi M, Amin M, Zamani M, Najdi D. Jundishapour J Microbiol Comparison of vancomycin and cefazolin therapeutic effect with povidone-Iodine on corneal ulcer in rabbits. 2012;5(3):491-5.

23. Deramo VA, Lai JC, Fastenberg DM, Udell IJ. Acute endophthalmitis in eyes treated prophylactically with gatifloxacin and moxifloxacin. Am J Ophthalmol. 2006;142(5):721-5. 